\title{
FATORES PREDITIVOS DE MORBIDADE E MORTALIDADE NO TRAUMA HEPÁTICO
}

\author{
PREDICTIVE FACTORS OF MORBIDITY AND MORTALITY IN HEPATIC TRAUMA
}

\author{
Alan César Diório; Gustavo Pereira Fraga, TCBC-SP²; Iêso Dutra Júnior ${ }^{3}$; \\ João Luís de Genova Corrêa Joaquim ${ }^{3}$; Mario Mantovani, ECBC-SP ${ }^{4}$
}

\begin{abstract}
RESUMO: Objetivo: Identificar os fatores preditivos de complicações e mortalidade em doentes operados com trauma hepático. Método: Estudo retrospectivo de 638 pacientes tratados no período de 1990 a 2003, identificando estatisticamente parâmetros epidemiológicos, fisiológicos e anatômicos associados com maior morbidade e mortalidade. Resultados: Trauma penetrante foi o mecanismo mais freqüente. A instabilidade hemodinâmica esteve presente em $21,1 \%$ das vítimas e o Índice de Gravidade das Lesões anatômicas (ISS) médio foi de 20,7. A maioria das lesões hepáticas foi grau III. A morbidade foi de $50,4 \%$, sendo as complicações relacionadas ao fígado mais freqüentes: sangramento persistente $(9,8 \%)$, abscesso intraperitoneal $(3,8 \%)$ e fístula biliar (3\%). As complicações não hepáticas ocorreram em 273 pacientes $(42,8 \%)$. A mortalidade foi de 22,1\% (141 casos) decorrente principalmente de sangramento persistente e sepse. As vítimas fatais apresentaram-se com pior índice fisiológico na admissão, com lesões hepáticas mais complexas e índices anatômicos mais graves quando comparadas aos sobreviventes. Conclusão: Os fatores preditivos de ocorrência de complicações hepáticas foram: idade maior que 60 anos, instabilidade hemodinâmica ou alteração de parâmetros fisiológicos na admissão, presença de lesões hepáticas complexas (grau > III) e índices anatômicos de gravidade de lesão abdominal (ATI) ou em outros segmentos corpóreos (ISS) elevados (= 25). Todas estas variáveis, mais a presença de lesões associadas abdominais e não abdominais e o mecanismo de trauma fechado foram preditivas de ocorrência de complicações não-hepáticas. Todos os fatores estudados, exceto a presença de lesões associadas abdominais, foram preditivos para a evolução a óbito (Rev. Col. Bras. Cir. 2008; 35(6): 397-405).
\end{abstract}

Descritores: Fígado/ferimentos e lesões; Traumatismos abdominais/complicações; Ferimentos e Lesões/mortalidade; Hemorragia; Laparotomia.

\section{INTRODUÇÃO}

O abdome é freqüentemente acometido nos traumatismos e o fígado, pelo seu tamanho e posição anatômica, é sede muito freqüente de lesões, tanto nos ferimentos penetrantes quanto nos traumatismos contu$\operatorname{sos}^{1-3}$.

Apesar dos avanços no atendimento inicial, da reanimação efetiva em excelentes centros de resgate, controle rápido do sangramento, evoluções nas técnicas operatórias e de manejos conservadores, além de suportes intensivos adequados, a mortalidade global dos traumatismos hepáticos mantém-se em torno de 5 a $27 \% \%^{4-9}$. Atualmente há uma tendência em se evitar as grandes ressecções e mobilizações hepáticas, prefere-se um tratamento cirúrgico menos agressivo, indica-se o controle de dano em pacientes instáveis, enquanto que em trauma contuso em paciente estável hemodinamicamente é priorizado o tratamento não-operató- rio e a angioembolização através de radiologia intervencionista, com sucesso terapêutico entre 85 e $95 \%$ das vezes ${ }^{10-12}$.

Além do tratamento específico adequado, da preocupação com a qualidade e uniformidade das condutas, inclusive com programas de treinamento e aperfeiçoamento das equipes envolvidas nestes atendimentos, e do grau da lesão observada (do ferimento hepático em si), inúmeros outros fatores contribuem e influenciam na morbidade e mortalidade. Fatores como a intensidade da perda sanguínea, presença de lesões associadas abdominais ou extra-abdominais e o volume de hemoderivados utilizados são também importantes ${ }^{7,9}$. A grande freqüência e influência destes fatores associados à lesão hepática exigem um conhecimento aprofundado de suas interferências na evolução destas vítimas. O estudo dos valores preditivos de má evolução destes elementos em uma amostra bem catalogada é de grande interesse.

Devido à importância do assunto e sua freqüência na prática cirúrgica diária, despontou interesse o estudo da

1. Médico Assistente do Serviço de Cirurgia do Hospital do Trabalhador, Curitiba - PR; Mestre em Cirurgia da FCM - Unicamp.

2. Professor Assistente Doutor da Disciplina de Cirurgia do Trauma da FCM - Unicamp; Pós-Doutorado na Universidade da Califórnia, San Diego, com bolsa FAPESP.

3. Médico e ex-aluno da Liga do Trauma da Disciplina de Cirurgia do Trauma da FCM - Unicamp.

4. Professor Titular e Chefe da Disciplina de Cirurgia do Trauma da FCM - Unicamp.

Recebido em 06/07/2008

Aceito para publicação em 16/09/2008

Conflito de interesse: nenhum

Fonte de financiamento: nenhuma

Trabalho realizado na Disciplina de Cirurgia do Trauma do Departamento de Cirurgia da Faculdade de Ciências Médicas (FCM) da Universidade Estadual de Campinas (Unicamp). 
evolução dessas vítimas. Graças à experiência acumulada pelo serviço com aplicação de protocolos nestes quatorze anos, o trabalho propôs estudar os pacientes com trauma hepático tratados cirurgicamente, analisando diferentes parâmetros, comparando-os estaticamente e estabelecendo quais fatores se mostraram importantes na evolução dos mesmos, no que se refere à morbidade e à mortalidade.

\section{MÉTODO}

O trabalho foi desenvolvido na Disciplina de Cirurgia do Trauma, que é a responsável pelo atendimento das urgências cirúrgicas traumáticas e não-traumáticas no Hospital de Clínicas (HC) da Unicamp, situado na cidade de Campinas, no interior do estado de São Paulo. O hospital é universitário, de nível de complexidade terciário e atende pacientes do Sistema Único de Saúde (SUS) da Região Metropolitana de Campinas, com população estimada em 2,4 milhões de habitantes.

Foram avaliados os prontuários e protocolos dos pacientes vítimas de trauma, operados no período de $1^{\circ}$ de janeiro de 1990 a 31 de dezembro de 2003. Neste período, a equipe de plantonistas da Disciplina de Cirurgia do Trauma do HC - Unicamp realizou 14.798 procedimentos cirúrgicos, dos quais 2.922 ocorreram em vítimas de trauma. Destes procedimentos, 2.279 (78\%) foram laparotomias nas quais, em 638 casos (28\%), foram observadas lesões hepáticas, isoladas ou em associação a outras lesões, abdominais ou não-abdominais. Pacientes submetidos à laparotomia associada a outros procedimentos, como toracotomia lateral ou esternotomia, foram incluídos no estudo. Os pacientes submetidos à toracotomia de reanimação na sala de urgência e encaminhados ao centro cirúrgico, foram incluídos, quando era feita a laparotomia e identificada a lesão no fígado, independente da presença de atividade cardíaca espontânea. Também participaram do estudo, traumatizados em que, inicialmente, foi indicado tratamento não-operatório de órgãos abdominais, e que, posteriormente, houve a necessidade de tratamento cirúrgico. Com a finalidade de estudar as lesões abdominais associadas ao trauma hepático e seus valores preditivos para a ocorrência de complicações, o diafragma foi considerado como órgão abdominal neste estudo.
Foram excluídos da análise os casos em que os pacientes foram atendidos e acompanhados exclusivamente com tratamento não-operatório, embora sabidamente apresentassem lesões hepáticas documentadas através de tomografia, e os que evoluíram a óbito sem terem sido encaminhados ao centro cirúrgico.

Os dados epidemiológicos analisados foram: gênero, idade, mecanismo de trauma (TF, trauma fechado; FPAF, ferimento por projétil de arma de fogo; FAB, ferimento por arma branca), instabilidade hemodinâmica na admissão (PAS, pressão arterial sistólica menor que $90 \mathrm{mmHg}$ no atendimento inicial), índice fisiológico (Escore de Trauma Revisado, ou Revised Trauma Score, ou RTS), gravidade das lesões anatômicas hepáticas (Organ Injury Scaling, da Associação Americana de Cirurgia do Trauma - Tabela 1) ${ }^{13}$, índices anatômicos (Índice de Gravidade da Lesão, ou Injury Severity Score, ou ISS; e Índice de Trauma Abdominal, ou Abdominal Trauma Index, ou ATI), lesões associadas abdominais e não abdominais, procedimentos cirúrgicos realizados para o tratamento das lesões hepáticas, morbidade relativa e não relativa ao fígado, índices mistos (TRISS ou Probabilidade de Sobrevida) e mortalidade, especificando as causas do óbito.

O RTS é calculado a partir da Escala de Coma de Glasgow (GCS), pressão arterial sistólica (PAS) e freqüência respiratória (FR), sendo o valor máximo de 7,84. O ISS quantifica a gravidade das lesões anatômicas em diferentes segmentos corpóreos, com valor máximo de 75, e doentes com ISS > 25 apresentam graves lesões anatômicas. O ATI é utilizado para quantificar o risco de complicações após traumatismo abdominal. Cada órgão intra-abdominal tem um fator de risco que varia de um a cinco e, em cada órgão, a lesão é pontuada de acordo com a gravidade, recebendo valores também de um a cinco. O escore de lesão de cada órgão é o produto do fator de risco pela gravidade da lesão neste órgão, e a soma dos escores de todos os órgãos lesados representa o ATI, sendo que doentes com ATI = 25 apresentam graves lesões abdominais. O TRISS representa, baseado em estudos estatísticos utilizando o RTS, ISS, idade do doente e menanismo de trauma (TF ou trauma penetrante) a probabilidade de sobrevida estimada para o traumatizado.

Tabela 1 - Classificação das lesões hepáticas.

\begin{tabular}{|c|c|c|}
\hline$\overline{\text { Grau }}$ & & Descrição da Lesão \\
\hline \multirow[t]{2}{*}{ I } & Hematoma & subcapsular, $<10 \%$ da superfície \\
\hline & Laceração & $<1 \mathrm{~cm}$ de profundidade no parênquima \\
\hline \multirow[t]{2}{*}{ II } & Hematoma & subcapsular entre 10 e $50 \%$ da superfície ou intra-parenquimatoso $<10 \mathrm{~cm}$ de diâmetro \\
\hline & Laceração & $1 \mathrm{a} 3 \mathrm{~cm}$ de profundidade no parênquima $<10 \mathrm{~cm}$ de comprimento \\
\hline \multirow[t]{2}{*}{ III } & Hematoma & $\begin{array}{l}\text { subcapsular }>50 \% \text { da superfície, ou expansivo, ou rôto intra-parenquimatoso }>10 \mathrm{~cm} \text {, } \\
\text { expansivo ou rôto }\end{array}$ \\
\hline & Laceração & $>3 \mathrm{~cm}$ de profundidade \\
\hline IV & Laceração & rotura do parênquima entre 25 a $75 \%$ de um lobo ou rotura de 1 a 3 segmentos num único lobo \\
\hline $\mathbf{V}$ & Laceração & rotura do parênquima $>75 \%$ de um lobo hepático ou de mais de 3 segmentos de um único lobo \\
\hline & Vascular & lesão de veia porta justa-hepática, ou veias hepáticas centrais, ou lesão de cava retro-hepática \\
\hline VI & Vascular & avulsão hepática \\
\hline
\end{tabular}


As lesões hepáticas foram corrigidas por procedimentos cirúrgicos menores (nenhum procedimento no fígado, cauterização, sutura simples e/ou sutura simples mais colocação de omento na área de sutura) ou maiores (oclusão temporária do pedículo hepático ou manobra de Pringle, desbridamento resseccional, colocação de balão intrahepático e/ou controle de dano). No caso de mais de uma lesão no fígado, com procedimentos menores e maiores realizados em associação, foram considerados como maiores.

As complicações relativas ao fígado foram assim definidas: ressangramento (mudança na evolução do doente com queda da pressão arterial ou hematócrito, necessitando de reoperação), fístula biliar (persistente por no mínimo sete dias, com volume de drenagem maior que $100 \mathrm{ml}$ por dia, necessitando de terapêutica endoscópica ou cirúrgica) ou infecção intraperitoneal (abscesso hepático ou intraperitoneal, tratados por drenagem percutânea ou relaparotomia). As complicações não-hepáticas foram caracterizadas por alteração da evolução da vítima e com quadro clínico, laboratorial ou exames de imagem que comprovassem ou levassem a suspeita diagnóstica, quer seja pulmonar, relativa ao sistema nervoso central, sepse ou insuficiência de múltiplos órgãos e sistemas (IMOS).

Os testes estatísticos usados foram: $t$ de Student, Qui-Quadrado ou exato de Fisher, de acordo com a natureza dos dados obtidos e objetivos do trabalho. O nível crítico para a rejeição da hipótese de nulidade, a partir do qual a diferença foi considerada como estatisticamente significativa, foi de 5\% ( $p<0,05)$, assinalando-se em negrito os valores estatisticamente significativos.

O trabalho foi aprovado pelo Comitê de Ética em Pesquisa com seres humanos da instituição.

\section{RESULTADOS}

Para os 638 pacientes, a média de idade foi de $28,9 \pm$ 10,8 anos, com mínima de 13 e máxima de 76 anos. A maior frequiência foi na faixa de 21 a 30 anos ( $40 \%$ dos casos). Houve predomínio do gênero masculino (553 casos - 86,7\%).

A maioria dos pacientes foi vítima de trauma penetrante (437 casos - 68,4\%), sendo que $299(46,8 \%)$ sofreram FPAF e $138(21,6 \%)$ FAB. Duzentos e um pacientes $(31,6 \%)$ foram vítimas de TF, sendo 103 casos de evento automobilístico, 57 de atropelamento, 15 de evento motociclístico e 26 de outros mecanismos de lesão.

Em relação à avaliação hemodinâmica na admissão hospitalar, 135 pacientes $(21,2 \%)$ apresentavam-se instáveis hemodinamicamente (PAS $<90 \mathrm{mmHg}$ ) enquanto que 503 $(78,8 \%)$ estavam estáveis. A freqüência de instabilidade hemodinâmica nos diferentes mecanismos de trauma (TF, FPAF e FAB) foi, respectivamente, $21,2 \%, 19,1 \%$ e $19,6 \%$, sem diferença estatisticamente significativa deste parâmetro nos pacientes dos diferentes grupos.

O RTS variou entre 0 a 7,84, com média de 7,02 $\pm 1,63$ na população estudada. A média do RTS variou conforme o mecanismo de trauma, sendo de $6,40 \pm 1,90$ no grupo TF, 7,27 $\pm 1,43$ no FPAF e 7,37 $\pm 1,31$ no FAB. A freqüência de pacientes com RTS alterado nos diferentes mecanismos de trauma foi de, respectivamente, 58,7\% (TF), 24,7\% (FPAF) e 21,7\% (FAB). Os pacientes com trauma fechado apresentaram RTS alterado com maior freqüência, o que foi estatisticamente significativo $(p<0,0001)$ se comparados aos pacientes com trauma penetrante. Não houve diferença significante no que se refere ao RTS (normal ou alterado), entre pacientes vítimas de FPAF e FAB.

Na laparotomia, observou-se que a maioria das vítimas (216 casos - 33,9\%) apresentava lesão hepática grau III e que em $85,9 \%$ dos casos foram encontradas lesões menores (graus I, II e III), conforme ilustrado na Figura 1.

Avaliando a gravidade das lesões nos diferentes mecanismos de trauma, observou-se que no TF, $18 \%$ das vítimas apresentavam lesão hepática complexa, sendo esta freqüência menor nos $\operatorname{FPAF}(12,8 \%)$ e FAB $(11,6 \%)$, embora sem diferença estatisticamente significante. A figura 2 ilustra as frequiências da gravidade das lesões hepáticas nos diferentes mecanismos de trauma. Nos 90 pacientes $(14,1 \%$ da amostra) com lesão hepática complexa, observou-se que a maioria deles (56 casos - 62,2\%) encontrava-se instável hemodinamicamente na admissão e com RTS alterado (53 casos - 58,9\%), o que foi estatisticamente significativo se comparado a pacientes com lesões menores $(p<0,001$ nos dois parâmetros avaliados).

Nestes pacientes operados, em 133 (20,8\%) não foi realizado nenhum procedimento para tratamento da lesão hepática, pois eram lesões menores, sem sangramento. Dez pacientes $(1,6 \%)$ evoluíram a óbito durante a laparotomia sem que fosse realizado qualquer procedimento terapêutico no fígado.

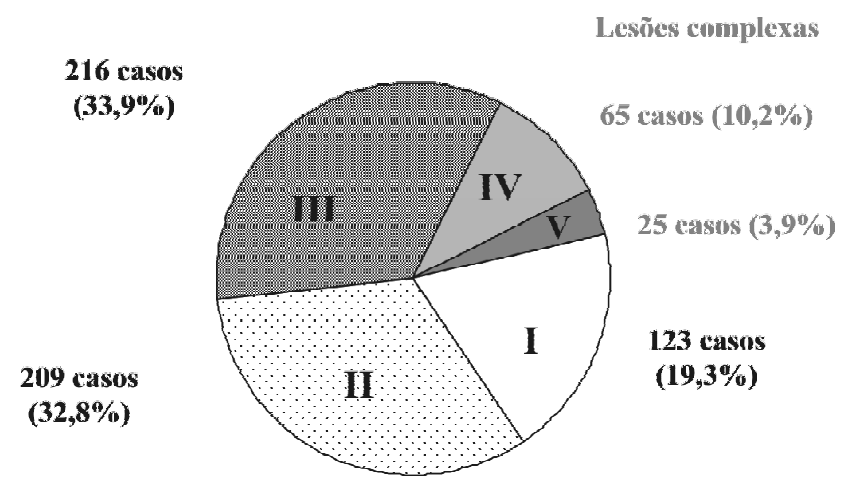

Figura 1 - Número de casos e porcentagem conforme a gravidade da lesão hepática.



Figura 2 - Porcentagem de pacientes conforme a gravidade da lesão hepática e o mecanismo de trauma. 
Nos demais 495 pacientes $(77,6 \%)$ foram realizados diferentes procedimentos, conforme apresentado na tabela 2. A sutura da lesão foi o procedimento realizado com maior frequiência (261 casos - 41,5\%). Além do procedimento cirúrgico para tratar a lesão, a drenagem da cavidade abdominal como procedimento complementar foi realizada em 322 pacientes $(51,3 \%)$.

Procedimentos menores foram realizados em $321 \mathrm{pa}-$ cientes (50,3\% da amostra total), com média de 1,1 procedimento por paciente. Considerando como procedimento menor os 133 casos que não necessitaram de tratamento da lesão hepática, obteve-se 454 casos $(71,2 \%)$ neste subgrupo. Os procedimentos maiores foram realizados em outros 174 casos $(27,3 \%)$, com média de 1,8 procedimento por paciente.

Analisando os procedimentos realizados nos pacientes vítimas de diferentes mecanismos de trauma, observouse que $32,1 \%$ (96 casos) dos FPAF necessitaram de procedimentos maiores, sendo estas porcentagens menores nos TF $(25,4 \%$ - 51 casos) e nas vítimas de FAB (20,6\% - 27 casos). Embora a porcentagem de lesões complexas tenha sido maior nas vítimas de trauma fechado (36 casos - 18\%), as vítimas de FPAF apresentaram uma elevada taxa de lesões hepáticas grau III (141 casos - 47,2\%), que exigiram procedimentos maiores para suas correções.

Lesões abdominais associadas foram identificadas em 482 casos $(75,5 \%)$, sendo mais freqüentes as de diafragma $(33,4 \%)$, estômago $(20,5 \%)$ e cólon / reto $(17,9 \%)$. Ocorreu um total de 977 lesões associadas abdominais nos 638 casos, com média de 1,5 lesões associadas ao fígado nestes pacientes. Nos TF foram mais freqüentes as lesões de baço $(24,9 \%)$ e nos penetrantes as de diafragma $(45,3 \%)$. Os números médios de lesões associadas abdominais, de acordo com o mecanismo de trauma (TF, FPAF e FAB) foram, respectivamente: 1,$0 ; 2,0$ e 1,1 .

Lesões associadas não-abdominais foram identificadas em 475 pacientes $(74,5 \%)$. Estas lesões ocorreram com maior freqüência em $\mathrm{TF}(87,6 \%$ dos pacientes neste subgrupo), se comparado a FPAF $(74,6 \%)$ e FAB $(55,1 \%)$. Nos TF houve predomínio de lesões nos segmentos craniencefálico (92 casos) e torácico ( 75 casos). Nos traumas penetrantes pre- dominaram as lesões de tórax (177 casos de FPAF e 58 casos de FAB).

O ATI teve uma média de $17,4 \pm 12,2$. Nos TF a média do ATI foi de 14,6 $\pm 8,6$, nos FPAF foi de 21,6 \pm 13,8 e nos FAB a média foi $12,5 \pm 10,1$. Na amostra, o ATI era maior que $25 \mathrm{em}$ 152 casos (23,8\%). Com relação aos mecanismos de trauma, os pacientes vítimas de FPAF apresentaram uma maior freqüência $(37,5 \%)$ de ATI > 25, o que foi estatisticamente significativo $(p<0,001)$, quando comparado aos outros mecanismos de trauma.

$\mathrm{Na}$ amostra, o ISS teve uma média de $20,7 \pm 11,4$. No grupo TF a média foi de $25 \pm 12,7$, nos FPAF foi de 20,5 $\pm 9,9$ e nos FAB foi de $14,7 \pm 9,6$. Analisando os pacientes com maior gravidade de lesão anatômica (ISS $=25$ ), observou-se maior frequiência de pacientes com TF $(49,8 \%)$, com valores estatisticamente significativos se comparados às vítimas de $\operatorname{FPAF}(p$ $<0,05)$ e de FAB $(p<0,001)$.

A morbidade geral na amostra estudada foi de 50,5\% (322 pacientes). As complicações relacionadas ao fígado ocorreram em 114 casos $(17,9 \%)$ e estão apresentadas na tabela 3 .

Os fatores preditivos de ocorrência de complicações hepáticas foram: idade maior que 60 anos; instabilidade hemodinâmica na admissão hospitalar; RTS alterado; presença de lesões hepáticas complexas; procedimentos cirúrgicos maiores para tratamento das lesões; ATI e ISS elevados (Tabela 4).

$\mathrm{Na}$ amostra, 273 pacientes $(42,8 \%)$ evoluíram com complicações não-hepáticas, sendo mais freqüentes as pulmonares ( 135 casos), sepse (62 casos), complicações relativas ao SNC (37 casos), choque hipovolêmico não reversível (85 casos) e três vítimas reoperadas devido à síndrome compartimental abdominal. Os fatores preditivos de ocorrência de complicações não-hepáticas foram todas as variáveis analisadas, incluindo o trauma fechado e a presença de lesões associadas, abdominais ou não.

A mortalidade na amostra foi de 22,1\% (141 casos), e as causas de óbito estão demonstradas na tabela 5 .

$\mathrm{Na}$ amostra estudada a média do TRISS foi de $0,89 \pm$ 0,24. A maioria dos pacientes ( 572 casos - 89,7\%) apresentou

Tabela 2 - Número de procedimentos cirúrgicos, menores e maiores, realizados no tratamento das lesões hepáticas.

\begin{tabular}{lcc}
\hline Procedimento Cirúrgico & Menor & Maior \\
\hline Nenhum & 133 & - \\
Cauterização & 164 & - \\
Sutura & 153 & 45 \\
Sutura com epíploon & 46 & 21 \\
Manobra de Pringle & - & 76 \\
Desbridamento resseccional menor & - & 21 \\
Desbridamento resseccional maior & - & 17 \\
Balão intrahepático & - & 45 \\
Manobra de Pachter & - & 15 \\
Sutura / ligadura de cava ou veias hepáticas & - & 3 \\
Shunt átrio-cava & - & 2 \\
Tamponamento com compressas (controle de danos) & - & 64 \\
Óbito antes do tratamento & - & 10 \\
\hline
\end{tabular}


Tabela 3 - Número de casos e porcentagem (sobre a amostra global) que evoluíram com complicações relacionadas à lesão do figado.

\begin{tabular}{lcc}
\hline Complicação & Número de Pacientes & Porcentagem \\
\hline Sangramento persistente / coagulopatia & 63 & 9,8 \\
Abscesso intra-abdominal & 24 & 3,8 \\
Fístula biliar & 19 & 3,0 \\
Coleperitônio & 10 & 1,6 \\
Abscesso hepático & 9 & 1,4 \\
Outras & 7 & 1,1 \\
\hline
\end{tabular}

TRISS maior que 0,50, e destes, 89 (15,5\%) evoluíram a óbito. Analisando as 141 vítimas fatais foram observadas as seguintes variáveis: média de idade de $32,5 \pm 0,8 ; 48,9 \%$ vítimas de trauma fechado; $52,5 \%$ destes pacientes foram admitidos instáveis hemodinamicamente; média do RTS de 5,35;32,6\% com lesões hepáticas complexas; $57,4 \%$ necessitaram de procedimentos cirúrgicos maiores; ATI médio de 24,7; ISS médio de
30,6; incidência de complicações hepáticas de 46,1\% e de nãohepáticas de 78\%; TRISS médio de 0,64.

Foram estudados diferentes fatores preditivos de mortalidade, conforme apresentado na tabela 6 . Todos foram, estatisticamente, preditivos para a evolução a óbito, exceto a presença de lesão associada abdominal, conforme a análise.

Tabela 4 - Número de casos e porcentagem conforme a presença de complicações hepáticas identificadas em diferentes subgrupos, com respectivas análises estatísticas.

\begin{tabular}{|c|c|c|c|}
\hline Variáveis/subgrupos & $\begin{array}{l}\text { Presença de complicações } \\
\text { (sim/não) }\end{array}$ & Freqüência de complicações & Valor de $p$ \\
\hline \multicolumn{4}{|l|}{$\overline{\text { Idade }}$} \\
\hline . $\leq 60$ anos & $109 / 517$ & $17,4 \%$ & \multirow[t]{2}{*}{$<0,05$} \\
\hline$. \quad>60$ anos & $5 / 7$ & $41,6 \%$ & \\
\hline \multicolumn{4}{|l|}{ Mecanismo de trauma } \\
\hline . fechado & $44 / 157$ & $21,9 \%$ & \multirow[t]{2}{*}{0,091} \\
\hline . penetrante & $70 / 367$ & $16,0 \%$ & \\
\hline \multicolumn{4}{|l|}{ PAS na admissão } \\
\hline . $\quad \geq 90 \mathrm{mmHg}$ & $65 / 438$ & $12,9 \%$ & \multirow[t]{2}{*}{$<0,001$} \\
\hline . $\quad<90 \mathrm{mmHg}$ & $49 / 86$ & $36,3 \%$ & \\
\hline \multicolumn{4}{|l|}{ RTS na admissão } \\
\hline . normal & $49 / 367$ & $11,8 \%$ & \multirow{2}{*}{$<0,001$} \\
\hline . alterado & $65 / 157$ & $29,2 \%$ & \\
\hline \multicolumn{4}{|c|}{ Gravidade da lesão hepática } \\
\hline · I a III & $62 / 486$ & $11,3 \%$ & \multirow[t]{2}{*}{$<0,001$} \\
\hline . IV ou V & $52 / 38$ & $57,8 \%$ & \\
\hline \multicolumn{4}{|l|}{ Procedimento cirúrgico } \\
\hline . menor & $35 / 419$ & $7,7 \%$ & \multirow[t]{2}{*}{$<0,001$} \\
\hline . maior & $71 / 103$ & $40,8 \%$ & \\
\hline \multicolumn{4}{|c|}{ Lesões abdominais associadas } \\
\hline . $\quad$ ausentes & $24 / 132$ & $15,3 \%$ & \multirow[t]{2}{*}{0,417} \\
\hline . $\quad$ presentes & $90 / 392$ & $18,6 \%$ & \\
\hline \multicolumn{4}{|c|}{ Lesões não-abdominais associadas } \\
\hline . ausentes & $24 / 139$ & $14,7 \%$ & \multirow{2}{*}{0,273} \\
\hline . $\quad$ presentes & $90 / 385$ & $18,9 \%$ & \\
\hline \multicolumn{4}{|l|}{ ATI } \\
\hline$\cdot \quad \leq 25$ & $70 / 416$ & $14,4 \%$ & \multirow[t]{2}{*}{$<0,001$} \\
\hline$\cdot \quad>25$ & $44 / 108$ & $28,9 \%$ & \\
\hline \multicolumn{4}{|l|}{ ISS } \\
\hline$\cdot \quad<25$ & $41 / 361$ & $10,2 \%$ & \multirow[t]{2}{*}{$<0,001$} \\
\hline . $\quad \geq 25$ & $73 / 163$ & $30,9 \%$ & \\
\hline
\end{tabular}


Tabela 5 - Número de casos e porcentagem das causas de óbito.

\begin{tabular}{lcc}
\hline Causa do Óbito & Número de Pacientes & Porcentagem \\
\hline Choque hipovolêmico & 76 & 54,0 \\
Sepse & 29 & 20,5 \\
IMOS & 16 & 11,3 \\
Trauma craniencefálico & 13 & 9,2 \\
Outros & 7 & 4,8 \\
Total & 141 & 100 \\
\hline
\end{tabular}

Tabela 6 - Número de casos e porcentagem conforme a mortalidade nos diferentes subgrupos, com respectivas análises estatísticas.

\begin{tabular}{|c|c|c|c|}
\hline Variáveis / Subgrupo & Óbitos (sim/não) & Mortalidade & Valor de $p$ \\
\hline \multicolumn{4}{|l|}{ Idade } \\
\hline . $\leq 60$ anos & $134 / 492$ & $21,4 \%$ & \multirow[t]{2}{*}{$<0,001$} \\
\hline . $>60$ anos & $7 / 5$ & $58,3 \%$ & \\
\hline \multicolumn{4}{|l|}{ Mecanismo de trauma } \\
\hline . fechado & $69 / 132$ & $34,3 \%$ & \multirow[t]{2}{*}{$<0,001$} \\
\hline . penetrante & $72 / 365$ & $16,5 \%$ & \\
\hline \multicolumn{4}{|l|}{ PAS na admissão } \\
\hline . $\quad \geq 90 \mathrm{mmHg}$ & $67 / 436$ & $13,3 \%$ & \multirow[t]{2}{*}{$<0,001$} \\
\hline . $\quad<90 \mathrm{mmHg}$ & $74 / 61$ & $54,8 \%$ & \\
\hline \multicolumn{4}{|l|}{ RTS na admissão } \\
\hline · normal & $36 / 380$ & $8,6 \%$ & \multirow[t]{2}{*}{$<0,001$} \\
\hline . alterado & $105 / 117$ & $47,3 \%$ & \\
\hline \multicolumn{4}{|c|}{ Gravidade da lesão hepática } \\
\hline · I a III & $95 / 453$ & $17,3 \%$ & \multirow[t]{2}{*}{$<0,001$} \\
\hline . $\quad$ IV ou V & $46 / 44$ & $51,1 \%$ & \\
\hline \multicolumn{4}{|l|}{ Procedimento cirúrgico } \\
\hline menor & $60 / 394$ & $13,2 \%$ & \multirow[t]{2}{*}{$<0,001$} \\
\hline . maior & $71 / 103$ & $40,8 \%$ & \\
\hline \multicolumn{4}{|c|}{ Lesões abdominais associadas } \\
\hline . ausentes & $26 / 130$ & $16,7 \%$ & \multirow[t]{2}{*}{0,07} \\
\hline . presentes & $115 / 367$ & $23,8 \%$ & \\
\hline \multicolumn{4}{|c|}{ Lesões não-abdominais associadas } \\
\hline . ausentes & $20 / 143$ & $12,3 \%$ & \multirow[t]{2}{*}{$<0,001$} \\
\hline . presentes & $121 / 354$ & $25,5 \%$ & \\
\hline \multicolumn{4}{|l|}{ ATI } \\
\hline . $\leq 25$ & $81 / 405$ & $16,7 \%$ & \multirow[t]{2}{*}{$<0,001$} \\
\hline.$>25$ & $60 / 92$ & $39,5 \%$ & \\
\hline \multicolumn{4}{|l|}{ ISS } \\
\hline$\cdot \quad<25$ & $38 / 364$ & $9,4 \%$ & \multirow[t]{2}{*}{$<0,001$} \\
\hline . $\quad \geq 25$ & $103 / 133$ & $43,6 \%$ & \\
\hline \multicolumn{4}{|c|}{ Complicações relativas ao fígado } \\
\hline . ausentes & $76 / 448$ & $14,5 \%$ & \multirow[t]{2}{*}{$<0,001$} \\
\hline . presentes & $65 / 49$ & $57,0 \%$ & \\
\hline \multicolumn{4}{|c|}{ Complicações não-hepáticas } \\
\hline . ausentes & $31 / 334$ & $8,5 \%$ & \multirow[t]{2}{*}{$<0,001$} \\
\hline . presentes & $110 / 163$ & $40,3 \%$ & \\
\hline
\end{tabular}

\section{DISCUSSÃO}

O trauma hepático corresponde a aproximadamente 5\% das admissões nas salas de urgência sendo a estrutura abdomi- nal mais freqüentemente lesada nas vítimas de traumatismos, com uma grande variação de aspectos relacionados às características de cada grupo de vítimas e ainda pelo fato de diferentes países e amostras comportarem aspectos epidemiológicos particulares ${ }^{2,3}$. 
Atualmente, existe uma tendência em tratar de modo não-operatório lesões mais complexas e até grupos selecionados de vítimas de ferimentos penetrantes ${ }^{14,15}$. Nos pacientes operados, grandes procedimentos sobre o fígado serão necessários em apenas $10 \%$ destas vítimas, e mesmo assim, com uma mortalidade superior a $30 \%$ nestes pacientes ${ }^{1,2,4,9,16,17}$.

Concordante com os estudos em nosso meio, o trabalho observou um número maior de vítimas de trauma penetrante ${ }^{18,19}$.

Nos diferentes mecanismos de trauma a instabilidade hemodinâmica apresentou semelhantes frequiências de aparecimento, todas próximas aos $20 \%$. Coimbra ${ }^{18}$, estudou 128 vítimas de traumatismo hepático penetrante e observou instabilidade hemodinâmica em $30 \%$ dos casos. O estudo relatando o acompanhamento e experiência de Brammer et $a l .^{20}$, em 2002, com 52 vítimas de trauma contuso, constatou instabilidade hemodinâmica em $23 \%$ dos casos, semelhante ao observado no presente trabalho. A presença de choque na admissão foi preditivo para a evolução com complicações relativas e não relativas ao fígado, bem como a óbito. Inúmeros trabalhos reforçam estes achados. Ao estudar os fatores que influenciaram a mortalidade de 44 vítimas operadas com lesões hepáticas graves, Chen et al. ${ }^{21}$ observaram uma mortalidade global de $70 \%$, sendo $50 \%$ dos casos decorrentes do trauma hepático. Nestas vítimas fatais, todos os pacientes que apresentavam PAS de zero na admissão morreram. A mortalidade no subgrupo que apresentava PAS $<90 \mathrm{mmHg}$ foi de $69 \%$ e nos indivíduos com PAS $=90 \mathrm{mmHg}$ foi de $37 \%$, reforçando a importância e a influência da estabilidade hemodinâmica na evolução destes doentes. Sikhondze et al. ${ }^{9}$, na experiência sul-africana de 105 doentes operados em sete anos apresentando trauma abdominal com lesão hepática, puderam observar que $58 \%$ das vítimas admitidas em choque hipovolêmico morreram e que, nas vítimas estáveis, a mortalidade foi $12 \%$.

Em Curitiba, estudando 200 vítimas com trauma hepático contuso, Von Bahten et al. ${ }^{19}$ observaram que $75 \%$ das vítimas apresentavam lesões hepáticas Graus I, II e III ("leves") e $25 \%$ lesões mais complexas (IV, V e VI). Em São Paulo, Coimbra observou uma taxa de mortalidade nas lesões menos graves (Grau I a III) de 6\% e de 50\% nas vítimas com lesões graves. Sikhondze et al. ${ }^{9}$ observaram progressiva mortalidade acompanhando o aumento da graduação da lesão hepática, a saber: Grau I, 11\%; Grau II, 16\%; Grau III, 22\%; Grau IV, 22\% e Grau V, $80 \%$. Kozar et al. ${ }^{16}$ correlacionaram o aumento da morbidade ao aumento da graduação da lesão hepática, sendo de $5 \%$ nas vítimas com lesões grau III, de $22 \%$ nas com lesões Grau IV e de $52 \%$ nas com Grau V. Scollay et al. ${ }^{7}$ também observaram a presença de lesões complexas em 30\% de 542 pacientes tratados em um período de 11 anos. Neste trabalho, as lesões mais graves foram acompanhadas do aumento do ISS e da mortalidade ${ }^{7}$. Cogbill et al. ${ }^{22}$ em estudo multicêntrico com 1.335 pacientes com trauma hepático, observaram uma mortalidade progressiva, de $26 \%$ nos pacientes com lesão grau III, de $46 \%$ nos com Grau IV e de $80 \%$ naqueles com lesões hepáticas Grau V.

Com relação aos procedimentos cirúrgicos realizados para a correção destas lesões, a sutura simples foi o procedimento cirúrgico mais utilizado nas vítimas que tiveram algum procedimento cirúrgico sobre o fígado (41,5\% dos procedimentos), o mesmo resultado observado em outros traba- lhos relativos às vítimas operadas com lesões hepáticas ${ }^{1,6,9,12,18}$ No presente trabalho, os procedimentos menores foram realizados em 71,2\% das vítimas, com uma média de 1,1 procedimentos por doente. Os procedimentos complexos foram utilizados em 27,3\% dos casos, com média de 1,8 procedimentos por doente. Estes procedimentos mais complexos foram utilizados com maior freqüência nas vítimas de $\operatorname{FPAF}(32,1 \%)$ que nas vítimas de trauma fechado e de FAB $(25,4 \%$ e $20,6 \%$, respectivamente). Interessante observar que apesar de um número maior de lesões complexas nas vítimas de traumatismo fechado, os procedimentos cirúrgicos mais complexos foram utilizados com maior freqüência nas vítimas de FPAF. Estes procedimentos cirúrgicos mais complexos correlacionaram-se com a evolução para complicações hepáticas, não-hepáticas e com a evolução para óbito. Inúmeros trabalhos correlacionam o aparecimento de altas taxas de mortalidade e de morbidade, secundárias especificamente à lesão hepática, à gravidade da lesão hepática e à dificuldade na correção das mesmas, traduzida pela utilização de procedimentos cirúrgicos mais complexos nestes $\operatorname{casos}^{1,7,9,21,23}$.

No presente estudo observou-se que a presença de lesões associadas abdominais foram preditivas apenas para a evolução para complicações não-hepáticas, sem correlação estatística entre estas e a evolução para complicações hepáticas e para óbito. Sikhondze et al. ${ }^{9}$, no estudo de 105 pacientes com traumatismo hepático, não observaram diferença estatística significativa na evolução para complicações nas vítimas com lesões associadas abdominais. Outros autores observaram outra realidade, onde a presença de lesões associadas no abdome (especialmente as de cólon, grandes vasos abdominais e pâncreas) interferiu negativamente na evolução dos doentes, com um maior número de complicações nestas vítimas que apresentavam lesões associadas ao ferimento hepático ${ }^{18,24}$.

Scollay et al. ${ }^{7}$ observaram que $90 \%$ dos doentes estudados tinham outras lesões não-hepáticas, abdominais ou não. Os autores também observaram que as vítimas de trauma fechado tinham mais lesões associadas do que as de trauma penetrante, e isto foi determinante para uma pior evolução, o que está de acordo com os achados do presente trabalho.

Velmahos et al. ${ }^{25}$ observaram que as vítimas que apresentavam ISS maior, apresentavam lesões hepáticas mais graves, maior necessidade de reposição volêmica, lesões associadas em outros aparelhos mais graves e que evoluíram com maior número de complicações e tempo mais prolongado de internação hospitalar. Outro estudo comparou o ISS com a morbidade e mortalidade: em doentes com ISS menor que nove a morbidade foi de $15 \%$ e a mortalidade de $2 \%$; com o ISS entre 10 e 20 , a morbidade $51 \%$ e a mortalidade $23 \%$; naquelas com ISS maior que 20 , a morbidade e a mortalidade mantiveram-se em torno de $63 \%$. Os achados destes estudos confirmam e reforçam a importância e a correlação deste índice anatômico com a evolução dos pacientes, em concordância aos nossos achados.

Das 638 vítimas estudadas, observou-se um total de 322 doentes que complicaram (morbidade de 50,4\%). Os fatores preditivos de evolução para complicações hepáticas foram: a idade maior que 60 anos, a instabilidade hemodinâmica na admissão, o RTS alterado, a presença de lesões hepáticas complexas e de procedimentos cirúrgicos também complexos 
utilizados nas suas correções, o ATI e o ISS elevados. Além destes fatores, o agente agressor trauma fechado e a presença de lesões associadas abdominais e não-abdominais foram preditivos de evolução para complicações não-hepáticas. As fístulas biliares no trauma de fígado ocorrem numa freqüência que varia de $0,5 \%$ a $20 \%$ 5,9,10,23,26. Sikhondze et al. ${ }^{9}$ observaram o aparecimento de complicações em $37 \%$ das vítimas, sendo que as complicações pulmonares ocorreram em $16 \%$ das mesmas, a IMOS em $13 \%$, a peritonite em $10 \%$, a infecção de parede em $6 \%$ e a fístula biliar em $6 \%$ dos mesmos. Não observaram diferença na morbidade na evolução das vítimas nos diferentes subgrupos de agentes agressores (FPAF, FAB e TF) e observaram que, quanto menor o ISS e quanto mais estável a vítima na admissão, menor era a morbidade encontrada .

Kozar et al..$^{16}$, estudando 699 vítimas com lesões hepáticas complexas, observaram que as complicações que ocorriam em até três dias de pós-operatório estavam relacionadas às complicações por sangramento persistente / coagulopatia e pela síndrome de compartimento abdominal (cinco doentes), e que a partir do terceiro dia, relacionavam-se com complicações infecciosas e biliares. Os autores também observaram que o grau da lesão hepática e o volume de reposição volêmica foram preditivos para maior incidência de complicações hepáticas ${ }^{16}$.

A mortalidade no presente estudo foi de $22,1 \%$. A mortalidade relativa ao traumatismo hepático vem diminuindo ao longo dos anos, graças aos recursos diagnósticos e terapêuticos menos invasivos disponíveis na atualidade, bem como a possibilidade de tratamento não-operatório e a realização do controle de dano auxiliado pela angioembolização, além de outros tantos recursos propedêuticos e terapêuticos disponíveis para o tratamento e condução destes casos, permitindo o decréscimo para uma mortalidade que atualmente se encontra em torno de $15 \%$. A mortalidade relativa ao fígado varia de acordo com o mecanismo de lesão, e vai de 1 a $40 \%$ nas diferentes séries, e dobra ou triplica na presença de lesões associadas abdominais ${ }^{2,3,27,28}$. Fabian et al ${ }^{27}$ observaram uma maior mortalidade em trauma fechado, não só pela tendência das lesões hepáticas serem mais graves, mas também e principalmente pela maior presença e gravidade de lesões associadas. Chen at $_{\text {al. }}{ }^{21}$, estudando 44 vítimas operadas apresentan- do lesão hepática complexa, observaram uma mortalidade de 68\% (30 óbitos). Para estas vítimas, os fatores preditivos de mortalidade foram: instabilidade hemodinâmica na admissão, diferença de base, escala de coma de Glasgow rebaixada, mecanismo de lesão (trauma fechado), número de segmentos hepáticos ressecados e total estimado de perda volêmica ${ }^{21}$.

A abordagem a vítimas de trauma hepático vem mudando paralelamente aos avanços e disponibilidade de recursos diagnósticos e terapêuticos. Estas mudanças poderão e deverão ser comparadas aos resultados que tratamentos anteriores obtiveram, visando confirmar ou não a melhoria da qualidade em termos de morbimortalidade que estes tratamentos proporcionam. Com o presente estudo conclui-se que os fatores preditivos para a ocorrência de complicações hepáticas foram a idade do paciente (> 60 anos), a instabilidade hemodinâmica na admissão, RTS alterado, presença de lesões hepáticas complexas com a realização de procedimentos cirúrgicos complexos para suas correções, e ATI e ISS elevados. Os valores preditivos para a ocorrência de complicações nãohepáticas foram todas as variáveis analisadas, inclusive o trauma fechado e a presença de lesões associadas abdominais e não abdominais. Todos os fatores, exceto a presença de lesões associadas abdominais, foram preditivos, estatisticamente, para a evolução a óbito. Estas vítimas apresentaram, ainda, piores índices de trauma e lesões hepáticas mais complexas, quando comparadas aos sobreviventes.

Neste sentido, o trabalho reflete uma casuística grande de pacientes tratados durante um longo período, traduzindo sua experiência e realidade. Além disso, abre caminho para uma série de trabalhos e pesquisas na área referente ao traumatismo hepático, já que os diferentes parâmetros estudados podem servir de base para outros estudos.

\section{AGRADECIMENTOS}

Os autores agradecem aos médicos: Elcio S. Hirano, Guilherme V. Meirelles, Jorge Carlos M. Curi, José Benedito Bortoto, LuizAntônio Albejante, Marcelo Pinheiro Villaça e Waldemar Prandi Filho, da Disciplina de Cirurgia do Trauma da Unicamp, que participaram da assistência aos pacientes do estudo.

\begin{abstract}
Background: The aim of this study was to evaluate predictive factors of morbidity and mortality in patients operated on for liver trauma. Methods: Six hundred thirty eight patients were studied from 1990 to 2003 regarding their epidemiologic, physiologic and anatomic data and the predictive values associated with complications and death were statistically analyzed. Results: Penetrating trauma was the most frequent mechanism of trauma. Hemodynamic instability occurred in $21.1 \%$ of the victims and mean Injury Severity Score (ISS) was 20.7. Most liver injuries were grade III. Morbidity was 50.4\% and the most common liver related complications were: persistent bleeding (9.8\%); intraperitoneal abscess (3.8\%) and biliary fistula (3\%). Complications not related to the liver occurred in 273 patients (42.8\%). Mortality was $22.1 \%$ (141 cases), and it occurred mainly due to persistent bleeding and sepsis. Fatal victims were more unstable during admission, with worst physiological status, more complex liver injuries and higher anatomical scores when in comparison with the other patients. Conclusion: We found that the predictive factors for liver complications were: age higher than 60 years-old, hemodynamic instability and altered physiological status during admission, complex liver injuries (grade > III), and higher anatomical scores (Abdominal Trauma Index and ISS = 25). All these variables, and the existence of associated abdominal and extra-abdominal injuries, and blunt mechanism of trauma were predictive of the occurrence of liver unrelated complications. All the studied factors, except for associated abdominal injuries, were predictive of death.
\end{abstract}

Key words: Liver/wounds \& injuries; Abdominal Injuries/complications; Wounds and Injuries/mortality; Hemorrhage; Laparotomy. 


\section{REFERÊNCIAS}

1. Pachter HL, Spencer FC, Hofstetter SR, Liang HG, Coppa GF. Significant trends in the treatment of hepatic trauma. Experience with 411 injuries. Ann Surg. 1992; 215(5):492-500; discussion 500-2.

2. Gür S, Orsel A, Atahan K, Hökmez A, Tarcan E. Surgical treatment of liver trauma (analyses of 244 patients). Hepatogastroenterology. 2003; 50(54):2109-11.

3. Coughlin PA, Stringer MD, Lodge JP, Pollard SG, Prasad KR, Toogood GJ. Management of blunt liver trauma in a tertiary referral centre. Br J Surg. 2004; 91(3):317-21.

4. Asensio JA, Demetriades D, Chahwan S, Gomez H, Hanpeter D, Velmahos G, Murray J, Shoemaker W, Berne TV. Approach to the management of complex hepatic injuries. J Trauma. 2000; 48(1):66-9.

5. Mohr AM, Lavery RF, Barone A, Bahramipour P, Magnotti LJ, Osband AJ, Sifri Z, Livingston DH. Angiographic embolization for liver injuries: Low mortality, high morbidity. J Trauma. 2003; 55:1071-81; discussion 1081-2.

6. Trunkey DD. Hepatic trauma: contemporary management. Surg Clin N Am. 2004; 84(2):437-50.

7. Scollay JM, Beard DR, Smith R, McKeown D, Garden OJ, Parks R. Eleven years of liver trauma: the Scottish experience. World J Surg. 2005; 29(6):744-9.=

8. Christmas AB, Wilson AK, Manning B, Franklin GA, Miller FB, Richardson JD, Rodriguez JL. Selective management of blunt hepatic injuries including nonoperative management is a safe and effective strategy. Surgery. 2005; 138(4):606-10; discussion 6101.

9. Sikhondze WL, Madiba TE, Naidoo NM, Muckart DJ. Predictors of outcome in patients requiring surgery for liver trauma. Injury. 2007; 38(1):65-70. Epub 2006 Nov 13.

10. Pachter HL, Knudson MM, Esrig B, Ross S, Hoyt D, Cogbill T, Sherman H, Scalea T, Harrison P, Shackford S, et al. Status of nonoperative management of blunt hepatic injuries in 1995: a multicenter experience with 404 patients. J Trauma. 1996; 40(1):31-8.

11. Ciraulo DL, Luk S, Palter M, Cowell V, Welch J, Cortes V, Orlando R, Banever T, Jacobs L. Selective hepatic arterial embolization of grade IV and V blunt hepatic injuries: an extension of resuscitation in the nonoperative management of traumatic hepatic injuries. J Trauma. 1998; 45(2):353-8; discussion 358-9.

12. David Richardson J, Franklin GA, Lukan JK, Carrillo EH, Spain DA, Miller FB, Wilson MA, Polk HC Jr, Flint LM. Evolution in the management of hepatic trauma: a 25 year perspective. Ann Surg. 2000; 232(3):324-30.

13. Moore EE, Cogbill TH, Jurkovich GJ, Sharkford SR, Malangoni MA, Champion HR. Organ injury scaling: spleen and liver (1994 revision). J Trauma. 1995; 38(3):323-4.

14. Kozar RA, Moore JB, Niles SE, Holcomb JB, Moore EE, Cothren CC, Hartwell E, Moore FA. Complications of nonoperative management of high-grade blunt hepatic injuries. J Trauma. 2005; 59(5):1066-71.

15. Demetriades D, Hadjizacharia P, Constantinou C, Brown C, Inaba K, Rhee P, Salim A. Selective nonoperative management of penetrating abdominal solid organ injuries. Ann Surg. 2006; 244(4):620-8.
16. Kozar AR, Moore FA, Cothren CC, Moore EE, Sena M, Bulger EM, Miller CC, Eastridge B, Acheson E, Brundage SI, Tataria M, McCarthy M, Holcomb JB. Risk factors for hepatic morbidity following nonoperative management. A multicenter study. Arch Surg. 2006; 141(5):451-8; discussion 48-9.

17. Stracieri LD, Scarpelini S. Hepatic injury. Acta Cir Bras. 2006; 21:(suppl 1): 85-8.

18. Coimbra RS. Ferimentos hepáticos penetrantes: estudo dos fatores relacionados à morbidade e mortalidade operatórias. [Dissertação]. São Paulo (SP): Faculdade de Ciências Médicas da Santa Casa de São Paulo; 1992.

19. Von Bathen LC, Nicoluzzi JE, Olandoski M, Pantanali CA, Silva RF. Trauma abdominal fechado: análise dos pacientes vítimas de trauma hepático em um Hospital Universitário de Curitiba. Rev Col Bras Cir. 2005; 32(6):316-20.

20. Brammer RD, Bramhall SR, Mirza DF, Mayer AD, McMaster $\mathrm{P}$, Buckels JA. A 10 years experience of complex liver trauma. $\mathrm{Br}$ J Surg. 2002; 89(12):1532-7.

21. Chen RJ, Fang JF, Lin BC, Hsu YP, Kao JL, Chen MF. Factors determining operative mortality of grade $\mathrm{V}$ blunt hepatic trauma. J Trauma. 2000; 49(5):886-91.

22. Cogbill TH, Moore EE, Jurkovich GJ, Feliciano DV, Morris J, Mucha P. Severe hepatic trauma: a multi-center experience with 1.335 liver injuries. J Trauma. 1988; 28(10):1433-8.

23. Wahl LW, Brandt MM, Hemmila MR, Arbabi S. Diagnosis and management of bile leaks after blunt liver injury. Surgery. 2005; 138(4):742-7; discussion 747-8.

24. Nance ML, Peden GW, Shapiro MB, Kauder DR, Rotondo MF, Schwab CW. Solid viscus injury predictis major hollow viscus injury in blunt abdominal trauma. J Trauma. 1997; 43(4):618-22; discussion 622-3.

25. Velmahos GC, Toutouzas K, Radin R, Chan L, Rhee P, Tillou A, Demetriades D. High success with nonoperative management of blunt hepatic trauma: The liver is a study organ. Arch Surg. 2003; 138(5):475-8; discussion 480-1.

26. Mantovani M. Complicações do tratamento cirúrgico do trauma hepático. Revista FCM/UNICAMP. 2002; 9(1):53-62.

27. Fabian TC, Croce MA, Stanford GG, Payne LW, Mangiante EC, Voeller GR, Kudsk KA. Factors affecting morbidity following hepatic trauma. A prospective analysis of 482 injuries. Ann Surg. 1991; 213(6):540-7; discussion 548.

28. Duane TM, Como JJ, Bochicchio GV, Scalea TM. Reevaluating the management and outcomes of severe blunt liver injury. $\mathrm{J}$ Trauma. 2004; 57(3):494-500.

Como citar este artigo:

Diório AC, Fraga GP, Dutra Jr I, Joaquim JL, Mantovani M. Fatores preditivos de morbidade e mortalidade no trauma hepático. Rev Col Bras Cir. [periódico na Internet] 2008; 35(6). Disponível em URL: http://www.scielo.br/rcbc

Endereço para correspondência:

Alan César Diório

Rua Coronel Vitorino Ordine, 2010

83005-040 - São José dos Pinhais - PR

E-mail: alandiorio@yahoo.com.br 